\title{
ON SOME NEW N-INDEPENDENT-VARIABLE DISCRETE INEQUALITIES OF THE GRONWALL TYPE
}

\author{
EN HAO YANG \\ Department of Mathematics \\ Jinan University \\ Guang Zhou, China \\ (Received June 26, 1985)
}

ABSTRACT. In this paper we shall establish some new discrete inequalities of the Gronwal1 type in $\mathrm{N}$-independent variables. They will have many applications for finite difference equations involving several independent variables and for numerical analysis. Their consequence for the case of $N=3$, generalizes all of the known theorems obtained by Pachpatte and Singare in [1]. An example, to which those results established in [1] are inapplicable, is given here to convey the usefulness of the results obtained.

KEY WORDS AND PHRASES. Inequalities of the Gronwall type, difference equations. 1980 AMS SUBJECT CLASSIFICATION CODES. 26D05, 26D10 and 26D15.

1. PRELIMINARIES. It is we11-known that the discrete inequalities of the Gronwall type played a vital role in the theory of finite difference equations and numerical analysis (see [2-8] and [9-12] and the references therein). Recently, Pachpatte and Singare [1] have established some new discrete inequalities in three independent variables. Discrete inequalities involving more than one independent variables are very useful in the study of many important problems concerning discrete versions of some partial differential and integral equations in several variables.

The aim of this paper is to obtain several $\mathrm{N}$-independent-variable discrete inequalities which extend all results obtained in [1] for the case of $N=3$. In what follows we shall make use of the following notations and definitions.

Let $N_{0}$ be the infinite set consisting of the integers $0,1,2, \ldots$, and we shall use the convention of writing

$$
\sum_{j \in Z} b_{j} \equiv 0, \quad \prod_{j \in Z} c_{j} \equiv 1,
$$

if $Z$ is the empty set. For simplicity, in the sequel we will denote $\left(x_{1}, x_{2}, \ldots, x_{n}\right) \in N_{0}^{n}$ by $x$, and $\left(x_{1}, x_{2}, \ldots, x_{j}\right),\left(x_{j}, x_{j+1}, \ldots, x_{n}\right)$, and $\left(x_{i}, x_{i+1}, \ldots, x_{k}\right)$ by $x_{j}, \tilde{x}_{j}$, and $\bar{x}_{i, k}$ respectively, here $i, k$ are integers from $1,2, \ldots, n$ with $1<k$. Further, we denote the multiple-summation symbol 


$$
\sum_{y_{j}=0}^{x_{j}-1} \sum_{y_{j+1}=0}^{x_{j+1}-1} \cdots \sum_{y_{k}=0}^{x_{k}-1} \text { by } \sum_{y, j}^{x, k}
$$

where $x_{i}, y_{i} \in N_{0}, j \leq i \leq k$, and $1 \leq j \leq k \leq n$. Moreover, we define

$$
\begin{aligned}
& \Delta u_{x_{j}}(x)=u\left({\underset{\sim}{x-1}}_{\left.j-x_{j}+1, \widetilde{x}_{j+1}\right)-u(x),}\right. \\
& \Delta u_{x_{j} x_{k}}^{2}(x)=\Delta u_{x_{j}}\left({\underset{\sim}{x-1}}_{k-1}, x_{k}+1, \tilde{x}_{k+1}\right)-\Delta u_{x_{j}}(x), \\
& \ldots \ldots \ldots \ldots \ldots \ldots \ldots \\
& \ldots \ldots \ldots \ldots \ldots \ldots \ldots \ldots
\end{aligned}
$$

and so on, where $x_{j}, x_{k}, \ldots$, are numbers from $N_{o}$, and $j, k, \ldots$, are integers from $1,2, \ldots, n$. We write also here that

$$
\Delta^{(r)} L(x) \triangleq \Delta L_{x_{1} x_{2} \ldots x_{r}}^{r}(x)
$$

for, any real-valued function $L(x)$ on $N_{0}^{n}$, here $1 \leq r \leq n, x \in N_{0}^{n}$. In addition, we shall define a class of functions on $\mathrm{N}_{0}^{\mathrm{n}}$ by

$$
K=\left\{f: f(x) \geq 0, \Delta^{(r)} f(x) \geq 0, r=1, \ldots, n-1, \text { and } \Delta^{(n)} f(x) \leq 0\right\} .
$$

It is obvious that the following properties are true:

(1) if $f(x) \in K$ and $c \geq 0$ is a real number, then $\operatorname{cf}(x) \in K$.

(2) If $\mathrm{f}(\mathrm{x}), \mathrm{g}(\mathrm{x}) \in \mathrm{K}$ then $\mathrm{f}(\mathrm{x})+\mathrm{g}(\mathrm{x}) \in \mathrm{K}$.

(3) all functions of the form

$$
x_{i_{1}}^{r_{1}} x_{i_{2}}^{r_{2}} \ldots x_{i_{k-1}}^{r_{k-1}} \quad(k=1,2, \ldots, n)
$$

are in the class $K$, here $r_{h} \geq 0$ and $i_{h}(h=1, \ldots, k-1)$ are integers with $1 \leq i_{1}<i_{2}<\ldots<i_{k-1} \leq n$.

2. LINEAR INEQUALITIES.

THEOREM 1. Let $u(x)$ and $p(x)$ be real-valued non-negative functions defined for $x \in N_{0}^{n}$, and let $f(x)$ be a real-valued positive and nondecreasing function in K. Suppose further that the discrete inequality

$$
u(x) \leq f(x)+\sum_{y, 1}^{x, n} p(y) u(y)
$$

is satisfied for all $x \in N_{0}^{n}$. Then we have the inequality

$$
u(x) \leqslant f\left(0, \tilde{x}_{2}\right) \prod_{y_{1}=0}^{x_{1}^{-1}}\left[1+G\left(y_{1}, \tilde{x}_{2}\right)+\sum_{y, 2}^{x, n} p(y)\right], \quad x \in N_{0}^{n}
$$


where

$$
\begin{aligned}
G(x)= & g_{1}\left(x_{1}, 0, \tilde{x}_{3}\right)+\sum_{y, 2}^{x, 2} g_{2}\left(x_{1}, y_{2}, 0, \tilde{x}_{4}\right)+\ldots+ \\
& \sum_{y, 2}^{x, n-2} g_{n-2}\left(x_{1}, \bar{y}_{2, n-2}, 0, x_{n}\right)+\sum_{y, 2}^{x, n-1} g_{n-1}\left(x_{1}, \bar{y}_{2, n-1}, 0\right),
\end{aligned}
$$

and herein

$$
\boldsymbol{G}_{k}\left({\underset{\sim}{x}}_{k}, 0, \tilde{x}_{k+2}\right)=\frac{\Delta^{(k)} f\left({\underset{\sim}{x}}_{k}, 0, \tilde{x}_{k+2}\right)}{f\left({\underset{\sim}{k}}_{k}, 0, \tilde{x}_{k+2}\right)}
$$

for $1 \leq \mathrm{k} \leq \mathrm{n}-1, \mathrm{x} \in \mathrm{N}_{\mathrm{0}}^{\mathrm{n}}$.

PROOF. We define a function $U(x)$ on $N_{0}^{n}$ by the right member of (2.1), so that by definition

$$
\begin{aligned}
& U\left({\underset{\sim}{x-1}}_{k-1}, 0, \tilde{x}_{k+1}\right)=f\left({\underset{\sim}{x}-1}_{k}, 0, \tilde{x}_{k+1}\right)>0, \\
& U\left({\underset{\sim}{x}-1}_{k-1}, x_{k}+1, \tilde{x}_{k+1}\right) \geq U(x)>0, x_{k} \in N_{0}, 1 \leq k \leq n .
\end{aligned}
$$

since $f(x)$ is nondecreasing.

Further, we can obtain from the definition of $U(x)$ that,

$$
\begin{aligned}
\Delta^{(n)} U(x) & =\Delta^{(n)} f(x)+p(x) u(x) \\
& \leq p(x) U\left({\underset{\sim}{n-1}}_{n-1}, x_{n}+1\right) .
\end{aligned}
$$

since $\Delta(n) f(x) \leq 0, p(x) \geq 0,(2.1)$, and (2.5). In view of the fact that

$$
\Delta^{(k)} U(x)=\Delta^{(k)} f(x)+\sum_{y, k+1}^{x, n} p\left({\underset{\sim k}{x}}_{y^{\prime}, \tilde{y}_{k+1}}\right) u\left({\underset{\sim}{x}}_{k}, \tilde{y}_{k+1}\right) \geq 0,
$$

is valid for $1 \leq k \leq n-1$ and $x \in N_{\circ}^{n}$. By applying (2.5) and (2.7), we derive from $(2.6)$

$$
\frac{\Delta^{(n-1)} U\left({\underset{\sim}{n-1}}_{n}, x_{n}+1\right)}{U\left(x_{n-1}, x_{n}+1\right)}-\frac{\Delta^{(n-1)} U(x)}{U(x)} \leq p(x) .
$$

Keeping $\underset{\sim}{x_{n-1}}$ fixed in $\left(2.6^{\prime}\right)$ set $x_{n}=y_{n}$ and sum over $y_{n}=0,1,2, \ldots, x_{n}-1$ to obtain the inequality

$$
\frac{\Delta^{(n-1)} U(x)}{U(x)} \leq g_{n-1}\left({\underset{\sim}{x} n-1}_{U}, 0\right)+\sum_{y, n}^{x, n} p\left({\underset{\sim}{x}, 1}_{n-1}, y_{n}\right),
$$


where $g_{n-1}$ is given by $(2.4)$. We may rewrite the last inequality as

$$
\begin{aligned}
& \frac{\Delta^{(n-2)} U\left(x_{n-2}, x_{n-1}+1, x_{n}\right)}{U\left({\underset{\sim}{n}}_{n-2}, x_{n-1}+1, x_{n}\right)}-\frac{\Delta^{(n-2)} U(x)}{U(x)} \leqslant \\
& \leq g_{n-1}\left({\underset{\sim}{n-1}}_{n-1}, 0\right)+\sum_{y, n}^{x, n} p\left({\underset{\sim}{x-1}}_{n, y_{n}}\right) \text {. }
\end{aligned}
$$

since (2.5) and (2.7). Keeping now $x_{n-2}$ and $x_{n}$ fixed in (2.9), set $x_{n-1}=y_{n-1}$ and sum over $y_{n-1}=0,1,2, \ldots, x_{n-1}-1$ to get the inequality

$$
\begin{gathered}
\frac{\Delta^{(n-2)} U(x)}{U^{\prime}(x)} \leq g_{n-2}\left({\underset{\sim}{x-2}}_{n-2}, 0, x_{n}\right)+\sum_{y^{\prime}, n-1}^{x, n-1} g_{n-1}\left({\underset{\sim}{n-2}}_{n-2}, y_{n-1}, 0\right) \\
+\sum_{y, n-1}^{x, n} p\left({\underset{\sim n}{n-2},}_{y_{n-1}}\right),
\end{gathered}
$$

here $g_{n-2}$ is given by (2.4). If $n-2>1$, then by using a similar argument as used above for $(2.8)$ to $(2.10)$, we can obtain

$$
\begin{aligned}
& \frac{\Delta^{(n-3)} U(x)}{U(x)} \leq g_{n-3}\left({\underset{\sim n}{n-3}}_{n}, 0, \tilde{x}_{n-1}\right)+\sum_{y, n-2}^{x, n-2} g_{n-2}\left({\underset{\sim}{n-3}}_{n-3}, y_{n-2}, 0, x_{n}\right) \\
& +\sum_{y, n-2}^{x, n-1} g_{n-1}\left({\underset{\sim}{x-3}}_{n}, \bar{y}_{n-2, n-1}, 0\right)+\sum_{y, n-2}^{x, n} p\left({\underset{\sim n}{x-3}}_{n}, \tilde{y}_{n-2}\right) .
\end{aligned}
$$

Continuing in this way then we obtain

$$
\frac{\Delta^{(1)_{U}(x)}}{U(x)} \leq G(x)+\sum_{y, 2}^{x, n} p\left(x_{1}, \tilde{y}_{2}\right),
$$

where $G(x)$ is defined by (2.3). Obviously, the last inequality can be rewritten as

$$
\frac{U\left(x_{1}+1, \tilde{x}_{2}\right)}{U(x)} \leq 1+G(x)+\sum_{y, 2}^{x, n} p\left(x_{1}, \tilde{y}_{2}\right) \text {. }
$$

Keeping $\tilde{x}_{2}$ fixed in (2.11), set $x_{1}=y_{1}$ and then substitute $y_{1}=0,1,2, \ldots$, $x_{1}-1$ successively in $(2.11)$, we then get

$$
\frac{U(x)}{U\left(0, \tilde{x}_{2}\right)} \leqslant \prod_{y_{1}=0}^{x_{1}-1}\left[1+G\left(y_{1}, \tilde{x}_{2}\right)+\sum_{y, 2}^{x, n} p(y)\right] \text {. }
$$

Thus the desired bound for $u(x)$ in (2.2) follows from (2.1), (2.5) and (2.12) immediately. 
EXAMPLE 1. Suppose that the discrete inequality

$$
v\left(x_{1}, x_{2}, x_{3}\right) \leq a+x_{2} x_{3}+x_{1}^{4} x_{2}+\sum_{y, 1}^{x, 3} Q\left(y_{1}, y_{2}, y_{3}\right) v\left(y_{1}, y_{2}, y_{3}\right)
$$

holds for all $\left(x_{1}, x_{2}, x_{3}\right) \in N_{0}^{3}$, where $a>0$ is a constant, $v$ and $Q$ are real-valued non-negative functions defined on $\mathrm{N}_{0}^{3}$. Then, by Theorem 1 here we have the nondecreasing function

$$
f\left(x_{1}, x_{2}, x_{3}\right)=a+x_{2} x_{3}+x_{1}^{4} x_{2}(>0) \in K,
$$

since the following conditions are satisfied

$$
\begin{aligned}
& \Delta^{(1)} f\left(x_{1}, x_{2}, x_{3}\right)=x_{2}\left(1+4 x_{1}+6 x_{1}^{2}+4 x_{1}^{3}\right) \geq 0, \\
& \Delta^{(2)} f\left(x_{1}, x_{2}, x_{3}\right)=1+4 x_{1}+6 x_{1}^{2}+4 x_{1}^{3}>0, \text { for }\left(x_{1}, x_{2}, x_{3}\right) \in N_{0}^{3} \\
& \Delta^{(3)} f\left(x_{1}, x_{2}, x_{3}\right)=0 .
\end{aligned}
$$

so that,

$$
\begin{gathered}
g_{1}\left(x_{1}, 0, x_{3}\right)=\frac{\Delta^{(1)} f\left(x_{1}, 0, x_{3}\right)}{f\left(x_{1}, 0, x_{3}\right)}=0, \\
g_{2}\left(x_{1}, x_{2}, 0\right)=\frac{\Delta^{(2)} f\left(x_{1}, x_{2}, 0\right)}{f\left(x_{1}, x_{2}, 0\right)}=\frac{1+4 x_{1}+6 x_{1}^{2}+4 x_{1}^{3}}{a+x_{1}^{4} x_{2}} .
\end{gathered}
$$

Hence we derive the desired bound on $v$ from $(*)$ such that

$$
\begin{aligned}
v\left(x_{1}, x_{2}, x_{3}\right) \leq\left(a+x_{2} x_{3}\right) \prod_{y_{1}=0}^{x_{1}-1}\left\{1+\sum_{y, 2}^{x_{2} 2} \frac{1+4 y_{1}+6 y_{1}^{2}+4 y_{1}^{3}}{a+y_{1}^{4} y_{2}}+\right. \\
\left.+\sum_{y, 2}^{x, 3} Q\left(y_{1}, y_{2}, y_{3}\right)\right\}, \text { for }\left(x_{1}, x_{2}, x_{3}\right) \in N_{0}^{3} .
\end{aligned}
$$

We note here that the above inequality (*) can not be treated by means of the known results established in [1].

THEOREM 2. Let $u(x), f(x), p(x)$ be the same as in Theorem 1 , and let $q(x)$ be a real-valued non-negative function defined for $x \in N_{0}^{n}$. Suppose that the inequality

$$
u(x) \leq f(x)+\sum_{y, 1}^{x, n} p(y)\left[u(y)+\sum_{z, 1}^{y, n} q(z) u(z)\right]
$$

is satisfied for all $x \in \mathrm{N}_{0}^{\mathrm{n}}$. Then we also have

$$
u(x) \leqslant F(x)+\sum_{y, j}^{x, n} p(y) f\left(0, \tilde{y}_{2}\right) \prod_{z_{1}}^{y_{1}-1}\left[1+G\left(z_{1}, \tilde{y}_{2}\right)+\sum_{z, 2}^{r, n}(r(z)+q(z))\right] \text {, }
$$


for all $x \in N_{0}^{n}$, here $G$ is the same as in above Theorem 1 , and $F(x)$ is given by

$$
\begin{aligned}
F(x)= & f\left(0, \tilde{x}_{2}\right)+\sum_{y, 1}^{x, 1} \Delta^{(1)} f\left(y_{1}, 0, \tilde{x}_{3}\right)+\sum_{y, 1}^{x, 2} \Delta^{(2)} f\left({\underset{\sim}{2}}_{2}, 0, \tilde{x}_{4}\right)+\ldots+ \\
& \sum_{y, 1}^{x, n-2} \Delta^{(n-2)} f\left(\underset{\sim}{y}{ }_{n-2}, 0, x_{n}\right)+\sum_{y, 1}^{x, n-1} \Delta^{(n-1)} f\left(y_{n-1}, 0\right) \cdot(2.15)
\end{aligned}
$$

PROOF. We define two functions $V(x)$ and $W(x)$ on $N_{0}^{n}$ by the right number of (2.13) and the following equality

$$
h(x)=v(x)+\sum_{y, 1}^{x, n} q(y) v(y)
$$

respectively, so that by the definitions: $(f(x)$ is nondecreasing)

$$
\begin{aligned}
& w\left({\underset{\sim}{x}}_{J-1}, 0, \tilde{x}_{J+1}\right)=v\left({\underset{\sim}{x}}_{J-1}, 0, \tilde{x}_{J+1}\right)=f\left({\underset{\sim}{x}}_{J-1}, 0, \tilde{x}_{j+1}\right)>0, \\
& \mathrm{v}\left(\mathrm{x}_{j-1}, \mathrm{x}_{\mathrm{J}}+1, \tilde{\mathrm{x}}_{\mathrm{j}+1}\right) \geq \mathrm{v}(\mathrm{x})>0 \text {, } \\
& w\left(x_{j-1}, x_{j}+1, \tilde{x}_{j+1}\right) \geq w(x)>0 \text {, }
\end{aligned}
$$

where $x_{j} \in N_{o}^{n}, j=1,2, \ldots, n$. In addition, we obtain here

$$
\begin{array}{rl}
\Delta^{(r)} v(x)=\Delta^{(r)} f(x)+\sum_{y, r+1}^{x, n} & p\left({\underset{\sim r}{x},}_{\tilde{y}_{r+1}}\right)\left[u\left(\underset{\sim r}{x}, \tilde{\underline{v}}_{r+1}\right)+\right. \\
& \left.+\sum_{z, 1}^{x, r} \frac{y, n}{z, r+1} q(z) u(z)\right],
\end{array}
$$

and

$$
\begin{gathered}
\Delta^{(r)_{W}(x)=\Delta^{(r)} v(x)+\sum_{y, r+1}^{x, n}} q\left({\left.\underset{\sim r}{x}, \tilde{r}_{r+1}\right) v\left(\underset{\sim r}{x}, \tilde{y}_{r+1}\right) .}_{\text {for } 1 \leq r \leq n, x \in N_{0}^{n}}\right.
\end{gathered}
$$

Letting $\quad r=n$ in the above (2.20) and using $\lrcorner^{(n)} f(x) \leq 0$ we then derive that

$$
\Delta^{(n)} v(x) \leq p(x) w(x), \quad x \in N_{0}^{n}
$$

since $p(x), q(x)$ are non-negative and $u(x) \leq v(x) \leq w(x)$. Now by (2.21) we obtain

$$
\begin{array}{r}
\Delta^{(n)} W(x) \leq p(x) h(x)+q(x) v(x) \leq[p(x)+q(x)] W(x), \\
\text { for } x \in \Lambda_{0}^{-n}
\end{array}
$$


since $q(x) \geq 0$ and $V(x) \leq W(x)$. It is clear from (2.17) that,

$$
\frac{\Delta^{(r)_{W}\left(x_{r}, 0, \tilde{x}_{r+2}\right)}}{W\left({\underset{\sim}{x}}_{r}, 0, \tilde{x}_{r+2}\right)}=\frac{\Delta^{(r)} v\left(x_{r}, 0, \tilde{x}_{r+2}\right)}{V\left(\underline{\sim}_{r}, 0, \tilde{x}_{r+2}\right)}=g_{r}\left({\underset{\sim}{x}}_{r}, 0, \tilde{x}_{r+2}\right),
$$

where $1 \leq r \leq n-1, x \in N_{0}^{n}, j=1,2, \ldots, n$, and $g_{r}$ is given by (2.4).

Now by following the same argument as used in the proof of Theorem 1 , and using $(2.17),(2.24)$, we get from $(2.23)$

$$
w(x) \leq f\left(0, \tilde{x}_{2}\right) \prod_{y_{1}=0}^{x_{1}-1}\left[1+G\left(y_{1}, \tilde{x}_{2}\right)+\sum_{y, 2}^{x_{,} n}(p(y)+q(y))\right] .
$$

Substituting this bound for $W(x)$ in (2.22), and then rewrite it as follows

$$
\Delta^{(n-1)} v\left(x_{n-1}, x_{n}+1\right)-\Delta^{(n-1)} v(x) \leq h(x)
$$

where the function $h(x)$ is defined by

$$
h(x)=p(x) f\left(0, \tilde{x}_{2}\right) \prod_{y_{1}=0}^{x_{1}-1}\left[1+G\left(y_{1}, \tilde{x}_{2}\right)+\sum_{y, 2}^{x_{1} n}(p(y)+q(y))\right] .
$$

Keeping $x_{n-1}$ fixed in the above $(2.26)$, set $x_{n}=y_{n}$ and sum over $y_{n}=0,1,2, \ldots, x_{n}-1$ to get the estimate

$$
\begin{aligned}
& \Delta^{(n-1)} V(x) \leq \Delta^{(n-1)} v({\underset{\sim}{x}(n-1}, 0)+\sum_{y, n}^{x, n} h\left({\underset{\sim}{x-1}}_{n-1}, y_{n}\right) \\
& =\Delta^{(n-1)} f\left({\underset{\sim 1}{x-1}}_{n-0}, 0\right)+\sum_{y, n}^{x, n} h\left({\left.\underset{w n-1}{x}, y_{n}\right)}^{x}\right.
\end{aligned}
$$

Keeping now ${\underset{\sim}{n-2}}_{n-x_{n}}$ fixed in the last inequality, set $x_{n-1}=y_{n-1}$ and sum over $y_{n-1}=0,1,2, \ldots, x_{n-1}-1$ to get the inequality

$$
\begin{aligned}
\Delta^{(n-2)} v(x) \leqslant & \Delta^{(n-2)} f\left({\underset{\sim}{x-2}}_{n-2}, 0, x_{n}\right)+\sum_{y, n-1}^{x, n-1} \Delta^{(n-1)} f\left({\underset{\sim}{n-2}}_{n-2}, y_{n-1}, 0\right) \\
& +\sum_{y, n-1}^{x, n} n\left({\underset{\sim}{x}, 2}_{n-2}, \tilde{y}_{n-1}\right) .
\end{aligned}
$$


Continuing in this way then we, obtain

$$
\begin{aligned}
\Delta^{(1)} v(x) \equiv & v\left(x_{1}+1, \tilde{x}_{2}\right)-v(x) \\
\leqslant & \Delta^{(1)} f\left(x_{1}, 0, \tilde{x}_{3}\right)+\sum_{y, 2}^{x_{2} 2} \Delta^{(2)} f\left(x_{1}, y_{2}, 0, \tilde{x}_{4}\right)+\ldots+ \\
& \sum_{y, 2}^{x, n-1} \Delta^{(n-1)} f\left(x_{1}, \bar{y}_{2, n-1}, 0\right)+\sum_{y, 2}^{x, n} h\left(x_{1}, \tilde{y}_{2}\right) .
\end{aligned}
$$

Keeping $\tilde{x}_{2}$ fixed in $(2.27)$, set $x_{1}=y_{1}$ and substitute $y_{1}=0,1,2, \ldots, x_{1}-1$ successively in $(2.27)$ to derive the bound for $V(x)$ such that

$$
v(x) \leq F(x)+\sum_{y, 1}^{x, n} h(y)
$$

since $\mathrm{V}\left(0, \tilde{\mathrm{x}}_{2}\right)=\mathrm{f}\left(0, \tilde{\mathrm{x}}_{2}\right)$, where $\mathrm{F}(\mathrm{x})$ is given by (2.15). Hence the desired bound in (2.14) follows from (2.13), (2.28), and the definitions of $V(x)$ and $h(x)$ immediately. Q.E.D.

REMARK 1. Letting $\mathrm{n}=3$ and $\mathrm{f}(\mathrm{x})=\mathrm{a}_{1}\left(\mathrm{x}_{1}\right)+\mathrm{a}_{2}\left(\mathrm{x}_{2}\right)+\mathrm{a}_{3}\left(\mathrm{x}_{3}\right)$ in above Theorems 1,2 , where $a_{j}: N_{0} \rightarrow(0, \infty), \Delta a_{j}(z) \geq 0$ for all $z \in N_{0}, j=1,2,3$, then we derive the Theorems 1,2 of [1] respectively.

3. A NONLINEAR GENERALIZATION.

THEOREM 3. Let $u(x), p(x)$, and $f(x)$ be the same as in above Theorem 1 , and let $u(x) \geq u_{0}$ be satisfied for all $x \in N_{0}^{n}$, where $u_{0}$ is a positive number. Let $W(z)$ be a real-valued continuous, positive and strictly increasing function defined on the interval $\left(u_{0}, \infty\right)$. Suppose further that the inequality

$$
u(x) \leq f(x)+\sum_{y, 1}^{x, n} p(y) w(u(y)) \text {. }
$$

holds for $x \in N_{0}^{n}$. Then for $0 \leq x \leq x$ (this is $0 \leq x_{i} \leq x_{i}, i=1,2, \ldots, n$ ) we also have the inequality

$$
\left.u(x) \leq K^{-1}\left\{K\left[f\left(0, \tilde{x}_{2}\right)\right]+\sum_{y_{2} 1}^{x_{,} 1}\left[G^{*}\left(y_{1}, \tilde{x}_{2}\right)+\sum_{z, 2}^{x_{2} n} p_{1}, \tilde{z}_{2}\right)\right]\right\},
$$

where $K^{-1}$ denotes the inverse of $K$ and

$$
K(r)=\int_{r_{0}}^{r} \frac{d s}{w(s)} \quad \text { for } r \geq r_{0}\left(\geq u_{0}\right) \text {, }
$$

and the function $G^{*}(x)$ is obtained from $G(x)$ by replacing all of its denominators $f\left(x_{j}, 0, \tilde{x}_{j+2}\right)$ by $W\left[f\left({\underset{\sim}{j}}_{j}, 0, \tilde{x}_{j+2}\right)\right]$ respectively, here $j=1,2, \ldots, n-1$. Here $x \in N_{0}^{n}$ 
is chosen so that the expression contained in the brackets $\{.$.$\} in (3.2)$ belongs to the domain of $\mathrm{K}^{-1}$ as long as $0 \leq \mathrm{x} \leq \mathrm{x}$.

PROOF. Define a function $R(x)$ on $N_{0}^{n}$ by the right member of (3.1), so that

$$
R\left({\underset{\sim}{x}}_{j-1}, 0, \tilde{x}_{j+1}\right)=f\left({\underset{\sim}{x}-1}_{j}, 0, \tilde{x}_{j+1}\right) \geq u\left({\underset{\sim}{x}}_{j-1}, 0, \tilde{x}_{j+1}\right) \geq u_{0},
$$

for $j=1,2, \ldots, n$, and

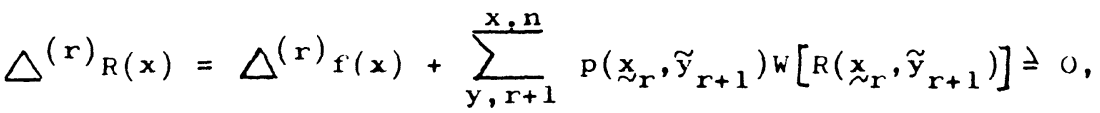

$$
\begin{aligned}
& 1 \leqslant r \leqslant n-1, x \in N_{0}^{n} \\
& \Delta^{(n)} R(x)=\Delta^{(n)} f(x)+p(x) w[u(x)] \leqslant p(x) \cdot w[R(x)], \quad x \in N_{0}^{n},
\end{aligned}
$$

since $\Delta^{(n)} f(x) \leq 0, p(x) \geq 0, u(x) \leq R(x)$, and $w(z)$ is increasing. We can observe from (3.6) that,

$$
\frac{\Delta^{(n-1)} R\left({\underset{\sim}{n-1}}_{n-1}, x_{n}+1\right)}{w\left[R\left({\underset{\sim n}{n-1}}_{n-1}, x_{n}+1\right)\right]}-\frac{\Delta(n-1)}{R(x)} \leq p(x),
$$

since (3.5), $R\left({\underset{\sim}{j}-1}_{j-1} x_{j}+1, \tilde{x}_{j+1}\right) \geq u_{o}$, and $W(z)$ is increasing. Using (3.4) and keeping $x_{n-1}$ fixed in (3.7), set $x_{n}=y_{n}$ and sum over $y_{n}=0,1,2, \ldots, x_{n}-1$ to derive the inequality

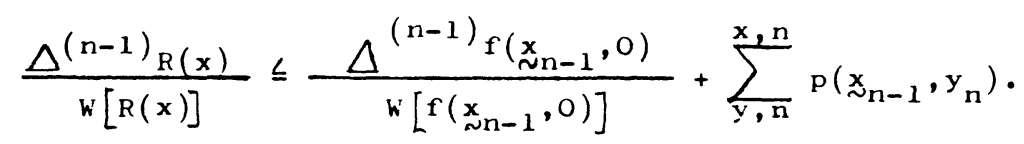

Replacing now the left member of (3.8) by the smaller term

$$
\frac{\Delta^{(n-2)} R\left(x_{n-2}, x_{n-1}+1, x_{n}\right)}{h\left[R\left({\underset{\sim n}{N n-2}}_{n-1}, x_{n-1}+1, x_{n}\right)\right]}-\frac{\Delta^{(n-2)} R(x)}{h[R(x)]},
$$

and then keeping ${\underset{\sim}{n-2}}_{n}$ and $x_{n}$ fixed in (3.8), set $x_{n-1}=y_{n-1}$ and sum over $\mathrm{y}_{\mathrm{n}-1}=0,1,2, \ldots, \mathrm{x}_{\mathrm{n}-1}-1$ to get the estimate

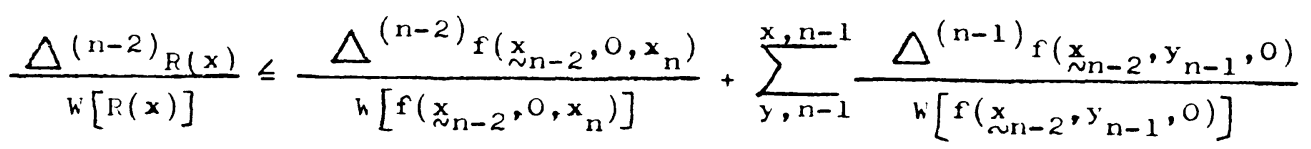

$$
\begin{aligned}
& +\sum_{y, n-1}^{x, n} p\left({\underset{N}{n-2}}_{n}, \tilde{y}_{n-1}\right) \text {. }
\end{aligned}
$$


Proceeding in this way we then obtain

$$
\frac{\Delta(1)_{R(x)}}{w[R(x)]} \leq G^{*}(x)+\sum_{y, 2}^{x, n} p\left(x_{1}, \tilde{y}_{2}\right) \quad x \in N_{o}^{n}
$$

where $G *(x)$ is obtained from $G(x)$ by the method as described in above. Obviously, (3.9) implies the following

$$
\begin{aligned}
K[R(\hat{x})]-K[R(x)] & =\int_{R(x)}^{R(\hat{x})} \frac{d s}{W(s)} \leq \frac{\Delta^{(1)} R(x)}{W[R(x)]} \\
& \leq G^{*}(x)+\sum_{Z: 2}^{x, n} P\left(x_{1}, \tilde{y}_{2}\right), \quad x \in N_{0}^{n}
\end{aligned}
$$

where $\hat{x}=\left(x_{1}+1, \tilde{x}_{2}\right)$. Keeping now $\tilde{x}_{2}$ fixed in the last inequality, set $x_{1}=y_{1}$ and sum over $y_{1}=0,1,2, \ldots x_{1}-1$ to obtain the inequality

$$
\left.K[R(x)]-K\left[R\left(0, \tilde{x}_{2}\right)\right] \leq \sum_{y, 1}^{x, 1}\left[G *\left(y_{1}, \tilde{x}_{2}\right)+\sum_{z, 2}^{x_{2} n} p\left(y_{1}, \tilde{z}_{2}\right)\right]\right], x_{0}^{n}
$$

and hence we have for $0 \leq \mathrm{x} \leq \mathrm{x}$

$$
R(x) \leqslant K^{-1}\left\{K\left[f\left(0, \tilde{x}_{2}\right)\right]+\sum_{y, 1}^{x, 1}\left[G^{*}\left(y_{1}, \tilde{x}_{2}\right)+\sum_{z, 2}^{x, n} p\left(y_{1}, \tilde{z}_{2}\right)\right]\right\}
$$

Hence the desired upper bound on $u(x)$ in (3.2) follows from (3.1), (3.10), and the definition of $R(x)$ immediately. The choice of $X \in N_{0}^{n}$ is obvious.

REMARK 2. By applying the same argument as used in the proofs of Theorems 2 and 3, we can easily establish an extension of the Theorem 4 of [1], which yields an upper bound for the solutions of the following inequality

$$
u(x) \leq f(x)+\sum_{y, 1}^{x, n} p(y)\left[u(y)+\sum_{z, 1}^{y, n} p(z) w^{\prime}(u(z))\right] .
$$

Here $x \in N_{0}^{n}$ and $u(x), f(x), p(x)$, and $W(z)$ are the same as defined in Theorem 3. Because the proof of this result is not difficult, so we leave it here to the reader. 


\section{REFERENCES}

1. PACHPATTE, B.G. and SINGARE, S.M. Discrete Generalized Gronwall Inequalities in Three Independent Variables, Pacific J. Math., 82 (1) (1979), 197-210.

2. BELLMAN, R. and COOKE, K.L. Differential-Difference Equations, Academic Press, New York, 1963.

3. BOPAEV, K.B. On Some Discrete Inequalities (Russian), Differencial'nye Uravnenja, Alma-Ata, $1981,35-44$.

4. DEMIdoviC, V.B. A Certain Criterion for the Stability of Difference Equations (Russian), Differencial'nye Uravnenija 5 (1969), 1247-1255.

5. BYKOV, Ja.V. and LINENKO, V.G. The Stability of the Solutions of Summary Difference Equations (Russian), Differencial'nye Uravnenija 9 (1973), 349-354.

6. JONES, G.S. Fundamental Inequalities for Discrete and Discontinuous Functional Equations, SIAM J. App1. Math. 12 (1964), 43-47.

7. MCKEE, S. Generalized Discrete Gronwall Lemmas, Z. Angew. Math. und Mech. 62 (9) (1982), 429-434.

8. PACHPATTE, B.G. Finite Difference Inequalities and Discrete Time Control Systems, Indian J. Pure App1. Math.9 (1978), 1282-1290.

9. POPENDA, J. and WERBOWSKI, J. On the Discrete Analogy of Gronwall Lemmas, Fas. Math. 11 (1979), 143-154.

10. SUGIYAMA, S. Difference Inequalities and Their Applications to Stability Problems, Lecture Notes in Mathematics 243 (1971), Springer, New York, 1-15.

11. WILLETT, D. and WONG, J.S.W. On the Discrete Analogues of Some Generalizations of Gronwall's Inequalities, Monatsh. Math. 69 (1964), 362-367.

12. YANG, E.H. On Some New Discrete Inequalities of the Bellman-Bihari Type, Nonlinear Anal.: TMA 7 (1983), 1237-1246. 


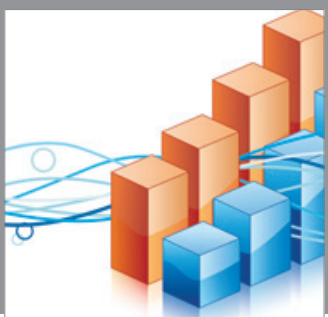

Advances in

Operations Research

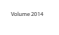

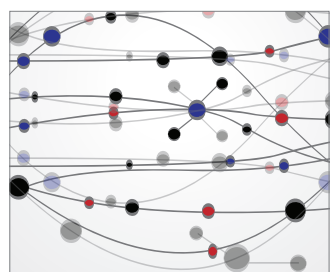

\section{The Scientific} World Journal
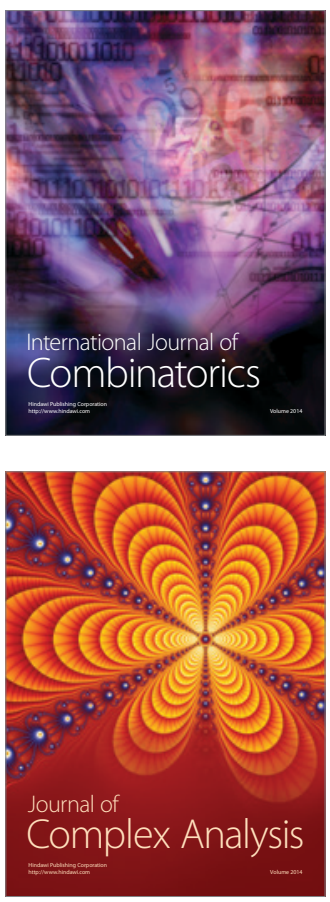

International Journal of

Mathematics and

Mathematical

Sciences
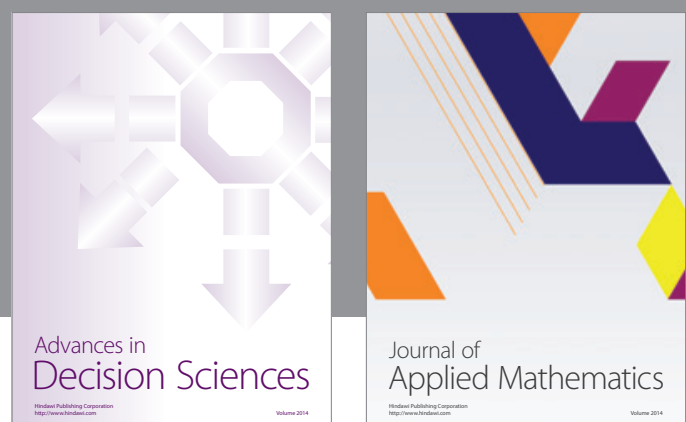

Journal of

Applied Mathematics
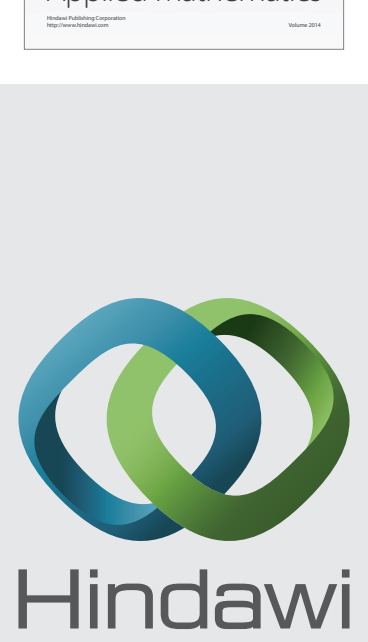

Submit your manuscripts at http://www.hindawi.com
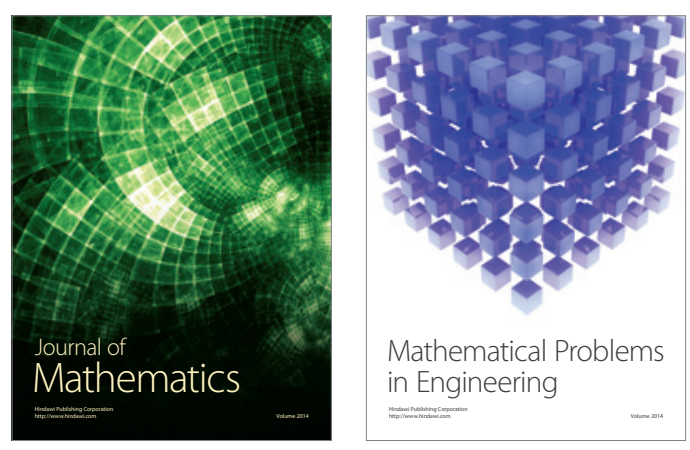

Mathematical Problems in Engineering
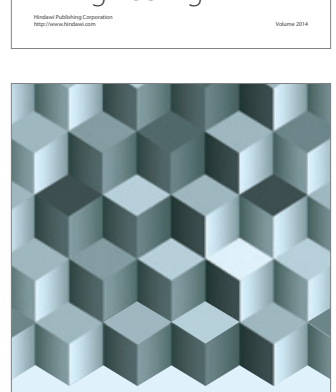

Journal of

Function Spaces
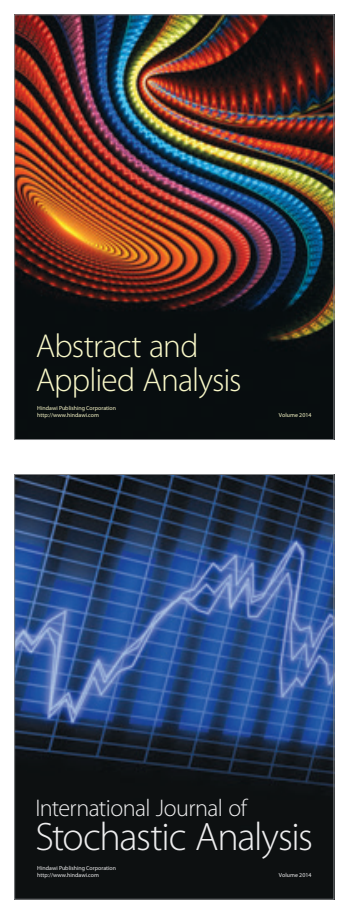

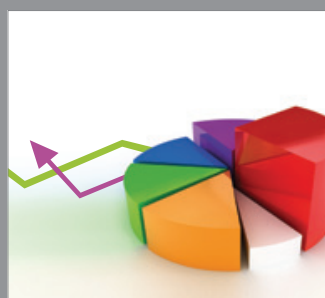

ournal of

Probability and Statistics

Promensencen
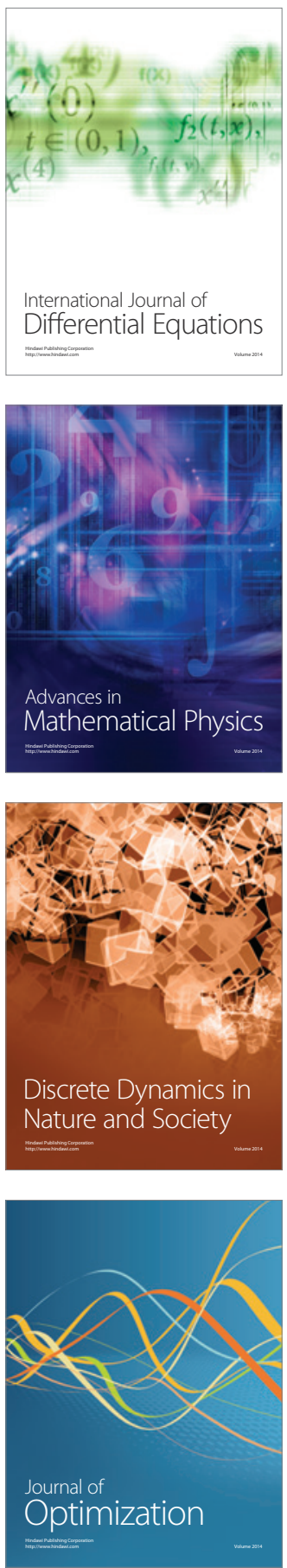